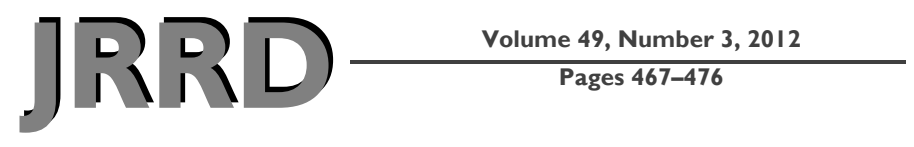

\title{
Accelerometer output and its association with energy expenditure in persons with multiple sclerosis
}

\author{
Brian M. Sandroff; Robert W. Motl, PhD; ${ }^{*}$ Yoojin Suh, MS \\ Department of Kinesiology and Community Health, University of Illinois at Urbana-Champaign, Urbana, IL
}

\begin{abstract}
Limited data support the strong association between rates of accelerometer activity counts and energy expenditure during dynamic activity in persons with multiple sclerosis (MS). This study examined the association between rates of activity counts and energy expenditure during walking by using two models of accelerometers and generated cut-points representing moderate-to-vigorous physical activity (MVPA) in persons with MS. Participants were 43 persons with MS and 43 controls who undertook 5 min of seated rest and up to five 6 min periods of walking at five different speeds on a treadmill. While walking, participants wore two models of accelerometers and a mouthpiece in-line with an open-circuit spirometry system for measuring energy expenditure (rate of oxygen consumption). Strong linear associations were found between accelerometer activity counts and energy expenditure, and the magnitude did not differ between MS and controls for both accelerometer models. The mean slopes of the linear relationships were steeper in persons with MS than controls and resulted in distinct cut-points for MVPA based on accelerometer counts for persons with MS and controls. The strong linear relationship between activity counts and energy expenditure and cut-points for quantifying time spent in MVPA should allow for better understanding of physical activity and examination of its predictors and consequences when using accelerometers in MS.
\end{abstract}

Key words: accelerometry, ActiGraph, activity counts, cutpoints, energetic cost, energy expenditure, multiple sclerosis, physical activity, treadmill, walking.

\section{INTRODUCTION}

Clinicians and researchers are increasingly interested in physical activity behavior among persons with multiple sclerosis (MS). This interest is based on physical activity's favorable association with symptom management, disease progression, and quality of life [1-3]. Accordingly, measurement is of importance in studying physical activity among persons with MS. In the event that the physical activity measurement does not accurately characterize the behavior, then the strength of the association between physical activity and symptom management, disease progression, and quality of life outcomes is likely to be underestimated or perhaps eliminated [4].

One approach for objectively measuring physical activity in persons with MS involves motion sensors (e.g., accelerometers). Accelerometers are typically worn on a belt around a person's waist (i.e., near the center of mass) and provide output of activity counts per unit time or epoch. The activity counts from the vertical axis of an accelerometer represent a digital integration of the positive and negative vertical displacement of the body's center of

Abbreviations: 7dPAR = 7-day physical activity recall, ANOVA $=$ analysis of variance, FAP $=$ Functional Ambulation Profile, MS = multiple sclerosis, MSWS-12 = 12-Item Multiple Sclerosis Walking Scale, MVPA = moderate-to-vigorous physical activity, PDDS = Patient-Determined Disease Steps, SD = standard deviation, $\mathrm{VO}_{2}=$ rate of oxygen consumption.

*Address all correspondence to Robert W. Motl, PhD; University of Illinois at Urbana-Champaign, Department of Kinesiology and Community Health, 233 Freer Hall, 906 South Goodwin Ave, Urbana, IL 61801; 217-265-0886; fax 217-244-7322. Email: robmotl@illinois.edu

http://dx.doi.org/10.1682/JRRD.2011.03.0063 
mass per unit time. This accelerometer output proportionally reflects the net external acceleration generated during bodily movement, and by extension, the output should be strongly associated with energy expenditure during physical activity. This assumption is consistent with the standard definition of physical activity as a behavior that involves bodily movement produced by contraction of skeletal muscles and resulting in increased energy expenditure above resting values [5].

The assumption of a strong association between accelerometer output and energy expenditure during dynamic physical activity [6-7] has been well tested in the general population [4,8-9], but not in persons with MS. This observation is important considering that the strength and slope of the association might be different in persons with MS than in the general population. Indeed, dynamic movements such as walking are associated with greater energy expenditure in persons with MS compared with controls [10-11]; no corresponding difference in accelerometer output exists [12]. Such a difference in energy expenditure, but not accelerometer output, would seemingly result in an association between activity counts and energy expenditure during dynamic activity that differs in persons with MS versus adults from the general population.

Further, examining the association between accelerometer output and energy expenditure during dynamic activity (i.e., walking) in persons with MS has a practical application. Researchers have calibrated accelerometer output and generated cut-points based on the rate of activity counts per minute that represent moderate-tovigorous physical activity (MVPA) for adults from the general population $[4,8]$. Such cut-points give the raw accelerometer output meaning by anchoring the activity counts with a biological substrate, namely energy expenditure. The cut-points have added valuable information for interpreting accelerometer output over and beyond total activity counts when physical activity in the general population of adults is being studied [9] and would be similarly informative for studying this behavior in persons with MS. Importantly, if the association between accelerometer output and energy expenditure during dynamic activity (i.e., walking) is different in persons with MS versus the general population, this would result in a cut-point for measuring time spent in MVPA that is specific for this population.

The present study examined the association between the rates of accelerometer activity counts and energy expenditure during the dynamic activity of walking in persons with MS. Such an examination will allow for a better understanding of physical activity behavior and its consequences in those with MS by testing a major assumption and providing cut-points for quantifying time spent in MVPA as an additional metric for interpreting accelerometer output. Walking was selected because it is the most common form of physical activity undertaken by persons with MS [13] and, by extension, represents a major source of overall physical activity energy expenditure. The primary objective of this study involved an examination of the association between the rates of activity counts and energy expenditure measured during walking in persons with MS compared with a control sample of adults without a chronic disease condition. The hypotheses were that (1) accelerometer activity counts and energy expenditure would both increase with walking speed for persons with MS and controls; (2) persons with MS would have greater energy expenditure than controls but similar accelerometer activity counts across a range of walking speeds; and (3) a strong linear relationship would exist between accelerometer activity counts and energy expenditure during walking in both samples, but the slope of the relationship would be steeper in persons with MS than controls. The secondary objective involved the calibration of accelerometer output for generating cut-points based on activity counts per minute that represented MVPA in persons with MS. We hypothesized that the cut-point for MVPA would be lower in persons with MS than controls.

\section{METHODS}

\section{Participants}

We recruited the sample of persons with MS through direct contact with support groups of a Midwestern chapter of the National Multiple Sclerosis Society that were located within an approximately 90 min drive of our campus. The sample of controls was recruited from within our campus and its surrounding community through public postings. The inclusion criteria for those with MS involved a clinically definite diagnosis of MS and no relapses during the past $30 \mathrm{~d}$ before testing. Additional inclusion criteria for all participants involved (1) being ambulatory without an assistive device, including cane, crutch, or walker; (2) being 18-64 yr of age; (3) having the visual ability necessary to read 14-point font; (4) being willing and able to wear the accelerometer and oxygen 
analysis system while walking on a treadmill; and (5) not having any risk factors for undertaking exercise testing based on the Physical Activity Readiness Questionnaire [14]. We screened 69 persons with MS and 49 controls; 20 persons with MS did not meet the inclusion criteria and 6 cancelled the testing sessions after screening, whereas 2 controls did not meet the inclusion criteria and 4 subsequently cancelled the testing sessions after screening. We enrolled final samples of 43 persons with MS and 43 controls matched on age, height, weight, and sex.

\section{Primary Measurements}

\section{Accelerometers}

The rates of activity counts were measured by ActiGraph model 7164 and model GT3X accelerometers (Health One Technology; Fort Walton Beach, Florida). We opted for two accelerometers because the model 7164 has most often been used in calibration studies with adults but is no longer commercially available, whereas the model GT3X is commercially available but has not been included in calibration studies. The model 7164 accelerometer is compact $(5.1 \times 4.1 \times 1.5 \mathrm{~cm})$ and lightweight (43 g) and contains a piezoelectric bender element on a cantilevered arm that generates an electrical signal proportional to the force acting on it. The model 7164 detects acceleration ranging in magnitude from $0.05-3.2 \mathrm{~g}$ and frequency ranging from $0.25-2.5 \mathrm{~Hz}$. Motion outside normal human movements is rejected by a band-pass filter. The acceleration signal is digitized by an 8-bit analog-todigital converter, with measurement amplitude of 0-256 units and a sampling rate of $10 \mathrm{~Hz}$, and is numerically integrated over a preset epoch interval. The integrated value is stored in memory as activity counts and the integrator is reset at the end of each interval. The data are retrieved from the accelerometer via a personal computer and reader interface unit and then imported into Microsoft Excel (Microsoft Corporation; Redmond, Washington) for processing.

The model GT3X accelerometer is small $(3.8 \times 3.7 \times$ $1.8 \mathrm{~cm}$ ) and lightweight (27 g) and contains a solid-state digital accelerometer that generates an electrical signal proportional to the force acting on it along three axes; this study only included the vertical axis for consistency with the model 7164. Acceleration detection for the model GT3X ranges in magnitude from $0.05-2.5 \mathrm{~g}$ and the frequency ranges from $0.25-2.5 \mathrm{~Hz}$, with motion outside normal human movements rejected by a band-pass filter. The acceleration signal is digitized by a 12-bit analog-to-digital converter, with measurement amplitude of 0-4,096 units and a sampling rate of $30 \mathrm{~Hz}$, and is numerically integrated over a preprogrammed epoch interval. The integrated value is stored in memory as activity counts and the integrator is reset at the end of each interval. The data are retrieved from the accelerometer via a direct USB (Universal Serial Bus) 2.0 connection with a personal computer and then imported into Microsoft Excel for processing.

Participants wore both accelerometers in-line on an elastic belt that was positioned on the nondominant hip (i.e., anterior axillary line at the waist level). We set the epoch to $15 \mathrm{~s}$ for flexibility during data analysis, and activity counts were expressed as the average counts per minute (counts $\cdot \mathrm{min}^{-1}$ ) across the 6 min periods of walking. The accelerometers were initially calibrated by the manufacturer before the onset of this study. This likely resulted in a precision with the ActiGraph accelerometers of 5 percent intermonitor variation and 2 percent intramonitor variation in controlled trials using a motorized turntable, per the manufacturer's standard [15].

\section{Energy Expenditure}

The rate of oxygen consumption $\left(\mathrm{VO}_{2}\right)$, or energy expenditure, was measured by breath-by-breath analysis using an open-circuit spirometry system (TrueOne, Parvo Medics; Sandy, Utah). The $\mathrm{O}_{2}$ and $\mathrm{CO}_{2}$ analyzers of the system were calibrated using room air and verified concentrations of gases. The flow meter was calibrated using a 3 L syringe (Hans Rudolph; Kansas City, Missouri), and while on the treadmill, participants breathed through a two-way non-rebreathable valve (Hans Rudolph, model $2700 \mathrm{~B}$; dead space $=95 \mathrm{~mL}$ ). Steady-state $\mathrm{VO}_{2}$ was expressed as the average $\mathrm{VO}_{2}$ value in milliliters per kilogram per minute across the final 3 min (minutes 4-6) of each 6 min period of walking.

\section{Protocol}

The protocol for this study was approved by a university institutional review board, and participants provided written informed consent. The protocol included two testing sessions that were separated by $7 \mathrm{~d}$. On the day of the first testing session, participants completed demographic and exercise history questionnaires, underwent a 7-day physical activity recall (7dPAR) [16], and then performed four trials of walking on a $26 \mathrm{ft}$ GAITRite mat (CIR Systems, Inc; Havertown, Pennsylvania) for measuring ambulatory ability based on the Functional Ambulation Profile (FAP) score 
[17]. Those with MS further completed the PatientDetermined Disease Steps (PDDS) scale [18] and 12Item Multiple Sclerosis Walking Scale (MSWS-12) [19] for characterizing the sample. We then measured each participant's height and weight with clothes by using a scalestadiometer unit (Detecto model 3P7044; Webb City, Missouri) and engaged the participant in an accommodation trial on the treadmill. The accommodation trial involved having the participant wear the accelerometers and oxygen analysis system while walking on a treadmill for 5-10 min across a range of speeds. This allowed for both practice with treadmill walking and determination of the appropriate range of speeds for the second session.

The second testing session was performed $7 \mathrm{~d}$ later. Participants initially were instrumented for the walking protocol and then sat quietly for $5 \mathrm{~min}$ to allow generation of an estimate of resting energy expenditure. The participants then undertook up to five 6 min periods of walking on a motor-driven treadmill (Trackmaster model TMX425C, Full Vision, Inc; Newton, Kansas) with 6 min periods of seated rest between the periods of walking. The five possible walking speeds were $54,67,80,94$, and $107 \mathrm{~m} \cdot \mathrm{min}^{-1}$ (i.e., slow through fast walking) and the actual speeds for the protocol among the persons with MS were determined from the accommodation trial. The order of speeds was further randomized for those with MS. The corresponding control participant completed the same speeds and in the same order as the MS participant. The treadmill speed was verified by measuring belt length and the time for 25 revolutions of the belt, and the treadmill grade $(0 \%)$ was verified by a digital inclinometer (Beall Tilt Box, The Beall Tool Company; Newark, Ohio).

\section{Data Processing and Analysis}

We entered the accelerometer and metabolic data per participant into Microsoft Excel. This allowed for computing the multiple correlation coefficient $(R)$, squared multiple correlation coefficient $\left(R^{2}\right)$, intercept, slope, and cut-point for MVPA (i.e., $\geq 3$ metabolic equivalents) based on a linear relationship between accelerometer activity counts and $\mathrm{VO}_{2}$ per participant. Importantly, we checked and confirmed the veracity of the output from Excel with PASW Statistics 18 (SPSS Inc; Chicago, Illinois).

The data analysis itself was done in PASW Statistics 18. The accelerometer and metabolic data recorded across the five possible treadmill speeds and the $R, R^{2}$, intercept, slope, and cut-point for MVPA for the two groups were analyzed with independent samples $t$-tests; the $t$-tests were one-tailed because of the directional hypothesis. We conducted $t$-tests on accelerometer activity counts and $\mathrm{VO}_{2}$ rather than a mixed model analysis of variance (ANOVA) given the unequal sample sizes across treadmill speeds (i.e., not all participants were capable of completing all five speeds); the ANOVA would have required complete data for all six points (i.e., seated rest and all five walking speeds), thereby restricting the sample size itself and possibly resulting in a unique sample of persons with highly intact walking capacity.

\section{RESULTS}

\section{Descriptive Statistics}

The sample consisted of 43 persons with a definite diagnosis of MS and 43 controls who were similar in age $(t(84)=0.31, p=0.76)$, sex $\left(\chi^{2}(1, n=84)=0.10, p=\right.$ $0.75)$, height $(t(84)=0.13, p=0.89)$, weight $(t(84)=0.06$, $p=0.95)$, physical activity levels from the 7dPAR $(t(84)=$ $0.16, p=0.88)$, exercise history $(t(84)=0.02, p=0.98)$, and ambulatory ability based on FAP scores $(t(84)=1.45$, $p=0.15)$. The descriptive characteristics are provided in Table 1. There were 39 persons with relapsing-remitting MS, 2 persons with secondary-progressive MS, and 2 persons with primary progressive MS. The mean \pm standard deviation (SD) duration of MS was $10.8 \pm 7.7 \mathrm{yr}$, and the median PDDS score was 1 (range $0-5$ ), indicating minimal disability (i.e., some noticeable but minor symptoms from MS with minimal effect on one's lifestyle). The mean \pm SD MSWS-12 score was $18.6 \pm 16.5$, further indicating minimal ambulatory impairment in those with MS.

Table 1.

Descriptive characteristics of participants with multiple sclerosis (MS) and controls.

\begin{tabular}{lcc}
\hline \multicolumn{1}{c}{ Variable } & MS $(\boldsymbol{n}=\mathbf{4 3})$ & Control $(\boldsymbol{n}=\mathbf{4 3})$ \\
\hline Age $(\mathrm{yr})$ & $47.2 \pm 9.1$ & $46.5 \pm 10.0$ \\
Sex $(n / \%$ female $)$ & $38 / 88.4$ & $38 / 88.4$ \\
Height $(\mathrm{cm})$ & $168.2 \pm 8.3$ & $168.5 \pm 8.9$ \\
Weight $(\mathrm{kg})$ & $75.7 \pm 19.4$ & $75.4 \pm 16.2$ \\
Physical Activity $(\mathrm{kcal} / \mathrm{wk})$ & $244.9 \pm 26.8$ & $245.7 \pm 15.5$ \\
Exercise History $(\mathrm{d} / \mathrm{wk})$ & $3.4 \pm 2.1$ & $3.4 \pm 1.9$ \\
FAP & $96.7 \pm 4.1$ & $95.1 \pm 5.8$ \\
\hline
\end{tabular}

Note: Values are reported as mean \pm standard deviation unless otherwise noted. FAP = Functional Ambulation Profile (from GAITRite electronic walkway). 


\section{Treadmill Protocol}

All 43 participants with MS completed the 5 min of resting energy expenditure and the 6 min periods of walking at speeds of 54 and $67 \mathrm{~m} \cdot \mathrm{min}^{-1}$. Only 35, 30, and 23 of the participants with MS completed the 6 min periods of walking at speeds of 80,94 , and $107 \mathrm{~m} \cdot \mathrm{min}^{-1}$, respectively.

\section{Accelerometer Data}

\section{Model 7164}

The accelerometer data from ActiGraph model 7164 are provided in Table 2. No differences were found between groups in accelerometer activity counts per minute for the speeds of $54 \mathrm{~m} \cdot \mathrm{min}^{-1}(t(84)=-0.23, p=$ $0.41), 67 \mathrm{~m} \cdot \mathrm{min}^{-1}(t(84)=0.18, p=0.43), 80 \mathrm{~m} \cdot \mathrm{min}^{-1}$ $(t(68)=0.31, p=0.38), 94 \mathrm{~m} \cdot \mathrm{min}^{-1}(t(58)=-0.21, p=$ $0.42)$, and $107 \mathrm{~m} \cdot \mathrm{min}^{-1}(t(44)=0.55, p=0.29)$.

\section{Model GT3X}

The accelerometer data from ActiGraph model GT3X are also provided in Table 2. Again, no differences were found between groups in accelerometer activity counts per minute for the speeds of $54 \mathrm{~m} \cdot \mathrm{min}^{-1}(t(84)=0.23, p=$ $0.41), 67 \mathrm{~m} \cdot \mathrm{min}^{-1}(t(84)=0.83, p=0.21), 80 \mathrm{~m} \cdot \mathrm{min}^{-1}$ $(t(68)=1.27, p=0.10), 94 \mathrm{~m} \cdot \mathrm{min}^{-1}(t(58)=0.67, p=$ $0.25)$, and $107 \mathrm{~m} \cdot \mathrm{min}^{-1}(t(44)=0.85, p=0.10)$.

\section{Metabolic Data}

The metabolic data are provided in Table 2 . No difference was found in resting metabolic rate between the two groups $(t(84)=-0.24, p=0.40)$. The persons with MS had higher energy expenditure than controls for the speeds of $54 \mathrm{~m} \cdot \mathrm{min}^{-1}(t(84)=-2.29, p=0.01)$,
$67 \mathrm{~m} \cdot \mathrm{min}^{-1}(t(84)=-3.02, p<0.01), 80 \mathrm{~m} \cdot \mathrm{min}^{-1}$ $(t(68)=-3.13, p<0.01)$, and $94 \mathrm{~m} \cdot \mathrm{min}^{-1}(t(58)=-2.00$, $p=0.02)$, but not $107 \mathrm{~m} \cdot \mathrm{min}^{-1}(t(44)=-0.89, p=0.19)$.

\section{Association Between Accelerometer and Metabolic Data}

\section{Model 7164}

A strong linear association existed between accelerometer activity counts and energy expenditure overall, with a mean \pm SD $R$ value of $0.96 \pm 0.03$ and $R^{2}$ value of $0.91 \pm 0.05$. The magnitude of the linear association did not differ between the samples of persons with MS and controls $(t(84)=-1.07, p=0.14)$, with mean \pm SD $R^{2}$ values of $0.91 \pm 0.05$ and $0.90 \pm 0.10$, respectively. The slope $(t(84)=-1.99, p=0.02)$, but not intercept $(t(84)=$ $-0.12, p=0.45$ ), of the linear relationship did differ between MS and controls, with a steeper mean \pm SD slope in MS of $0.003891 \pm 0.00222$ than in controls of $0.003136 \pm 0.00121$.

\section{Model GT3X}

A strong linear association existed between accelerometer activity counts and energy expenditure overall, with a mean \pm SD $R$ value of $0.93 \pm 0.04$ and $R^{2}$ value of $0.87 \pm 0.08$. The magnitude of the linear association did not differ between the samples of persons with MS and controls $(t(84)=-0.38, p=0.35)$, with mean $\pm \mathrm{SD} R^{2}$ values of $0.87 \pm 0.08$ and $0.86 \pm 0.13$, respectively. The slope $(t(84)=-1.61, p=0.05)$, but not intercept $(t(84)=$ $-0.84, p=0.20$ ), of the linear relationship did differ between MS and controls; there was a steeper mean \pm SD slope in MS of $0.003881 \pm 0.00213$ than in controls of $0.003223 \pm 0.00157$.

Table 2.

Metabolic data (rate of oxygen consumption $\left[\mathrm{VO}_{2}\right]$ ) and accelerometer data (ActiGraph models 7164 and GT3X, Health One Technology; Fort Walton Beach, Florida) for sitting and five speeds of treadmill walking in persons with multiple sclerosis (MS) and controls.

\begin{tabular}{|c|c|c|c|c|c|c|c|}
\hline \multirow{2}{*}{ Group } & \multirow{2}{*}{ Variable } & \multirow{2}{*}{ Sitting } & \multicolumn{5}{|c|}{ Speed $\left(\mathrm{m} \cdot \mathrm{min}^{-1}\right)$} \\
\hline & & & 54 & 67 & 80 & 94 & 107 \\
\hline \multirow[t]{3}{*}{$\overline{\mathrm{MS}}$} & $\mathrm{VO}_{2}$ & $3.7 \pm 0.6$ & $10.8 \pm 1.4$ & $12.3 \pm 1.7$ & $13.7 \pm 1.5$ & $15.7 \pm 1.3$ & $17.9 \pm 1.7$ \\
\hline & 7164 & $0 \pm 0$ & $1,276 \pm 509$ & $1,971 \pm 708$ & $2,936 \pm 916$ & $4,060 \pm 1,440$ & $4,716 \pm 1,544$ \\
\hline & GT3X & $0 \pm 0$ & $1,085 \pm 600$ & $1,845 \pm 758$ & $2,833 \pm 893$ & $3,803 \pm 1,199$ & $4,501 \pm 226$ \\
\hline \multirow[t]{3}{*}{ Controls } & $\mathrm{VO}_{2}$ & $3.7 \pm 0.6$ & $10.1 \pm 1.5$ & $11.3 \pm 1.5$ & $12.5 \pm 1.5$ & $14.8 \pm 2.0$ & $17.3 \pm 2.8$ \\
\hline & 7164 & $0 \pm 0$ & $1,254 \pm 382$ & $1,995 \pm 526$ & $2,999 \pm 739$ & $3,993 \pm 1,010$ & $4,940 \pm 1,205$ \\
\hline & GT3X & $0 \pm 0$ & $1,111 \pm 462$ & $1,969 \pm 624$ & $3,087 \pm 771$ & $3,994 \pm 988$ & $4,773 \pm 1,087$ \\
\hline
\end{tabular}

Note: Values are reported as mean \pm standard deviation. $\mathrm{VO}_{2}$ expressed as $\mathrm{mL} \cdot \mathrm{kg}^{-1} \cdot \mathrm{min}^{-1}$ and accelerometer data as counts $\cdot \mathrm{min}^{-1}$. 


\section{Cut-Points for Moderate-to-Vigorous Physical Activity}

\section{Model 7164}

The difference in the slope of the association between energy expenditure and accelerometer activity counts between samples resulted in different cut-points for MVPA: 1,723 \pm 732 counts $\cdot \mathrm{min}^{-1}$ in MS and 2,017 \pm 801 counts $\cdot \mathrm{min}^{-1}$ in controls.

\section{Model GT3X}

Again, the difference in the slope of the association between energy expenditure and accelerometer activity counts between samples resulted in different cut-points for MVPA: $1,584 \pm 697$ counts $\cdot \mathrm{min}^{-1}$ in MS and 1,950 \pm 852 counts $\cdot \mathrm{min}^{-1}$ in controls.

\section{DISCUSSION}

This study examined the association between rates of accelerometer output and energy expenditure during the dynamic activity of walking in persons with MS and controls. Such an examination was necessary for testing the assumption of a strong linear association between rates of activity counts and energy expenditure in MS and, in turn, deriving cut-points for quantifying time spent in MVPA as part of the output for interpreting free-living accelerometry. The primary novel results were that (1) energy expenditure, but not activity counts, differed between persons with MS and controls walking at speeds between 54 and $94 \mathrm{~m} \cdot \mathrm{min}^{-1}$; (2) a linear association existed between activity counts and energy expenditure in the overall sample and persons with and without MS, but the association was steeper in MS than controls; and (3) the cut-point for MVPA was lower in persons with MS than controls. This indicates that accelerometer output reflects energy expenditure during bodily movements such as walking and is consistent with the standard definition of physical activity (i.e., bodily movement from the contraction of skeletal muscles resulting in increased energy expenditure) [5]. Collectively, the results support the application of accelerometers along with cut-points for quantifying time spent in overall physical activity and MVPA among persons with MS. This is important for future research that will more precisely characterize the amount of physical activity and MVPA necessary for consequences such as symptom management, quality of life, and disease progression in MS. Such data will ultimately allow for better prescription and monitoring of physical activity within home- and clinic-based programs for maximizing the associated consequences in persons with MS.

One of the most important contributions of this research was the provision of cut-points for quantifying time spent in MVPA based on the rates of accelerometer activity counts per minute among persons with MS. Indeed, we identified cut-points for the model 7164 and GT3X accelerometers of 1,723 and 1,584 counts $\cdot \mathrm{min}^{-1}$, respectively, in the sample with MS. Those cut-points were lower than the values for controls of 2,017 and 1,950 counts'min ${ }^{-1}$ for the model 7164 and GT3X accelerometers, respectively. The difference in cut-points between samples was based on (1) the difference in energy expenditure, but not accelerometer output, between samples and (2) the steeper slope of the association between energy expenditure and accelerometer output in MS versus controls. Importantly, the MVPA cut-points for the model 7164 and GT3X accelerometers of 2,017 and 1,950 counts $\cdot \mathrm{min}^{-1}$, respectively, in the controls were remarkably similar to the value of 1,951 counts $\cdot \mathrm{min}^{-1}$ reported in the seminal research on calibration of accelerometers in nondisabled adults [4]. This is important because it provides evidence of consistency in both the methods and outcomes of our research with that of previous seminal research for providing MVPA cut-points for accelerometer data in controls and further exemplifies the observation that the cut-points for persons with MS are less than that of nondisabled controls, regardless of the model of accelerometer. This should be taken into consideration for subsequent research comparing physical activity between MS and controls, because the difference in MVPA cut-points might better reflect actual difference in physical activity between populations.

Across four of the five treadmill speeds, energy expenditure differed such that those with MS had a higher energetic cost of walking than controls. This was particularly prevalent with the slow-to-moderate walking speeds of 54, 67, and $80 \mathrm{~m} \cdot \mathrm{min}^{-1}$; those speeds were undertaken by more than 80 percent of the persons with MS and controls. Energy expenditure differed less with the speed of $94 \mathrm{~m} \cdot \mathrm{min}^{-1}$ and was no different with the speed of $107 \mathrm{~m} \cdot \mathrm{min}^{-1}$; those speeds were undertaken by fewer of the participants with MS and controls. There are several points of consideration for such results. We observed a difference in energy expenditure between MS and controls, despite the similarity of the samples on height, weight, physical activity, exercise history and, particularly, gait. This is interesting, because physical activity and exercise 
history as well as spatial and temporal parameters of gait are putative factors that influence the energetic cost of walking. Perhaps the difference in energetic cost of walking reflects more subtle consequences of MS that are not captured by other measures, particularly gait assessments of the spatial and temporal parameters of walking. The second observation is that many people with MS might not be capable of undertaking fast walking speeds on a treadmill, and perhaps, a slower speed might still reflect the disease-specific burden of MS for the energetic cost of walking. Indeed, a post-hoc analysis indicated that MSWS-12 scores were significantly correlated with the energetic cost of walking on a treadmill at the slowest walking speed of $54 \mathrm{~m} \cdot \mathrm{min}^{-1}(r=0.39, p=0.01)$. We believe that the energetic cost of slow walking may have merit as an outcome for capturing subtle changes in walking efficiency over time in MS.

A body of research has examined the validity and reliability of accelerometers in persons with MS, but seldom has the research included both persons with MS and controls. For example, researchers have reported that activity counts over a $7 \mathrm{~d}$ period from an accelerometer correlate with other measures of physical activity, namely self-report surveys, interview recalls, and motion sensors such as pedometers, in samples of persons with MS [2023]. Those studies generally have not examined the strength of the associations in persons with MS and controls for comparison of generalizability or specificity between populations. We further note that those studies have not tested one of the most important assumptions underlying application of an accelerometer: that the output itself reflects energy expenditure associated with bodily movement accumulated through physical activity. To that end, this study significantly advances the field by demonstrating a strong association between the rates of accelerometer activity counts and energy expenditure in both persons with MS and controls. By extension, this study further provides cut-points for directly quantifying time spent in MVPA for persons with MS that can be compared with nondisabled populations and included for further characterizing the association between physical activity and outcomes in persons with MS.

The study has several strengths, including the sample size and inclusion of controls and two models of accelerometers, but some important limitations should be considered when interpreting the results of this research. One limitation is that the sample mostly consisted of women with relapsing-remitting MS who had a short disease dura- tion and minimal disability. This was necessary given the constraints of the methodology, but limits the application of our results more broadly among those with progressive and advanced MS. We further measured accelerometer activity counts and energy expenditure while participants walked on a treadmill, so our results might not reflect overground walking and might be biased by degree of comfort with treadmill walking. We note that previous research has reported similar oxygen cost of overground and treadmill walking in MS [24], and we purposefully included an accommodation trial for providing practice with treadmill walking such that the novelty did not unduly influence our results. Another limitation is that we analyzed the data with independent samples $t$-tests, as opposed to a mixed model ANOVA. We performed $t$-tests rather than a mixed-model ANOVA because data were missing with increases in treadmill speed (i.e., some participants were unable to walk at faster speeds) and the ANOVA would have required complete data across all walking speeds. The limitation of our approach is the increased likelihood of a type-II error, but the problem with the ANOVA would be an increased likelihood of a type-I error based on a limited sample size.

\section{CONCLUSIONS}

The primary novel results were that (1) energy expenditure, but not accelerometer activity counts, differed between persons with MS and controls walking at speeds between 54 and $94 \mathrm{~m} \cdot \mathrm{min}^{-1}$; (2) a strong linear association existed between activity counts and energy expenditure in the overall sample and in persons with and without MS, but the association was steeper in MS than controls; and (3) the cut-point for MVPA from both models of accelerometer was lower in persons with MS than controls. This indicates that accelerometer output reflects energy expenditure during bodily movements such as walking and is consistent with the standard definition of physical activity [5]. Collectively, the results support the application of accelerometers along with cut-points for quantifying overall physical activity and time spent in MVPA, respectively, among persons with MS. This is essential for better characterizing the amounts of overall physical activity and MVPA that are necessary for maximizing consequences such as symptom management, quality of life, and disease progression in MS. Such data will allow for better prescription and monitoring of physical activity within 
home- and clinic-based programs for maximizing consequences in persons with MS.

\section{ACKNOWLEDGMENTS}

\author{
Author Contributions: \\ Study concept and design: B. M. Sandroff, R. W. Motl. \\ Acquisition of data: B. M. Sandroff, R. W. Motl, Y. Suh. \\ Analysis and interpretation of data: B. M. Sandroff, R. W. Motl. \\ Drafting of manuscript: B. M. Sandroff, R. W. Motl. \\ Critical revision of manuscript for important intellectual content: \\ B. M. Sandroff, R. W. Motl, Y. Suh. \\ Obtained funding: R. W. Motl.
}

Financial Disclosures: The authors have declared that no competing interests exist.

Funding/Support: This material was based on work supported by the Foundation of the Consortium of Multiple Sclerosis Centers.

Additional Contributions: We thank Swathi Balantrapu, Jenna Lungaro, Katie Stover, Anna Hoban, Jason Silberman, and Jennifer Medler for their assistance in data acquisition. Dr. Motl is also a consultant for Biogen IDEC and Acorda Therapeutics.

Institutional Review: This study was approved by the University of Illinois at Urbana-Champaign Institutional Review Board. During the study visit, written informed consent was obtained from each participant.

Participant Follow-Up: The authors plan to inform participants of the publication of this study.

\section{REFERENCES}

1. Heesen C, Romberg A, Gold S, Schulz KH. Physical exercise in multiple sclerosis: Supportive care or a putative disease-modifying treatment. Expert Rev Neurother. 2006; 6(3):347-55. [PMID:16533139] http://dx.doi.org/10.1586/14737175.6.3.347

2. Motl RW, Gosney JL. Effect of exercise training on quality of life in multiple sclerosis: A meta-analysis. Mult Scler. 2008; 14(1):129-35. [PMID:17881388] http://dx.doi.org/10.1177/1352458507080464

3. Snook EM, Motl RW. Effect of exercise training on walking mobility in multiple sclerosis: A meta-analysis. Neurorehabil Neural Repair. 2009;23(2):108-16. [PMID:18948413] http://dx.doi.org/10.1177/1545968308320641

4. Freedson PS, Melanson E, Sirard J. Calibration of the Computer Science and Applications, Inc. accelerometer. Med Sci Sports Exerc. 1998;30(5):777-81.

[PMID:9588623] http://dx.doi.org/10.1097/00005768-199805000-00021

5. Bouchard C, Blair SN, Haskell WL. Why study physical activity and health? In: Bouchard C, Blair Sn, Haskell WL, editors. Physical activity and health. Champaign, IL: Human Kinetics; 2007. p. 3-20.
6. Montoye HJ, Washburn R, Servais S, Ertl A, Webster JG, Nagle FJ. Estimation of energy expenditure by a portable accelerometer. Med Sci Sports Exerc. 1983;15(5):403-7. [PMID:6645869] http://dx.doi.org/10.1249/00005768-198315050-00010

7. Servais SB, Webster JG, Montoye HJ. Estimating human energy expenditure using an accelerometer device. J Clin Eng. 1984;9(2):159-70.

8. Leenders NY, Nelson TE, Sherman WM. Ability of different physical activity monitors to detect movement during treadmill walking. Int J Sports Med. 2003;24(1):43-50. [PMID:12582951] http://dx.doi.org/10.1055/s-2003-37196

9. Mathews CE. Calibration of accelerometer output for adults. Med Sci Sports Exerc. 2005;11:S512-22. http://dx.doi.org/10.1249/01.mss.0000185659.11982.3d

10. Olgiati R, Jacquet J, Di Prampero PE. Energy cost of walking and exertional dyspnea in multiple sclerosis. Am Rev Respir Dis. 1986;134(5):1005-10. [PMID:3777662]

11. Franceschini M, Rampello A, Bovolenta F, Aiello M, Tzani P, Chetta A. Cost of walking, exertional dyspnoea and fatigue in individuals with multiple sclerosis not requiring assistive devices. J Rehabil Med. 2010;42(8):719-23.

[PMID:20809053]

http://dx.doi.org/10.2340/16501977-0600

12. Motl RW, Snook EM, Agiovlasitis S. Does an accelerometer accurately measure steps taken under controlled conditions in adults with mild multiple sclerosis? Disabil Health J. 2011;4(1):52-57. [PMID:21168808] http://dx.doi.org/10.1016/j.dhjo.2010.02.003

13. Weikert M, Dlugonski D, Balantrapu S, Motl RW. Most common types of physical activity self-selected by people with multiple sclerosis. Int J MS Care. 2011;13(1):16-20.

14. Thomas S, Reading J, Shephard RJ. Revision of the Physical Activity Readiness Questionnaire (PAR-Q). Can J Sport Sci. 1992;17(4):338-45. [PMID:1330274]

15. Metcalf BS, Curnow JS, Evans C, Voss LD, Wilkin TJ. Technical reliability of the CSA activity monitor: The EarlyBird Study. Med Sci Sports Exerc. 2002;34(9):1533-37. [PMID:12218751] http://dx.doi.org/10.1097/00005768-200209000-00022

16. Sallis JF, Haskell WL, Wood PD, Fortmann SP, Rogers T, Blair SN, Paffenbarger RS Jr. Physical activity assessment methodology in the Five-City Project. Am J Epidemiol. 1985;121(1):91-106. [PMID:3964995]

17. Givon U, Zeilig G, Achiron A. Gait analysis in multiple sclerosis: Characterization of temporal-spatial parameters using GAITRite functional ambulation system. Gait Posture. 2009; 29(1):138-42. [PMID:18951800] http://dx.doi.org/10.1016/j.gaitpost.2008.07.011

18. Hadjimichael O, Kerns RD, Rizzo MA, Cutter G, Vollmer T. Persistent pain and uncomfortable sensations in persons 
with multiple sclerosis. Pain. 2007;127(1-2):35-41. [PMID:16949751]

http://dx.doi.org/10.1016/j.pain.2006.07.015

19. Hobart JC, Riazi A, Lamping DL, Fitzpatrick R, Thompson AJ. Measuring the impact of MS on walking ability: The 12-Item MS Walking Scale (MSWS-12). Neurology. 2003; 60(1):31-36. [PMID:12525714]

20. Kos D, Nagels G, D’Hooghe MB, Duquet W, Ilsbroukx S, Delbeke S, Kerckhofs E. Measuring activity patterns using actigraphy in multiple sclerosis. Chronobiol Int. 2007; 24(2):345-56. [PMID:17453852] http://dx.doi.org/10.1080/07420520701282364

21. Motl RW, McAuley E, Snook EM, Scott JA. Validity of physical activity measures in ambulatory individuals with multiple sclerosis. Disabil Rehabil. 2006;28(18):1151-56. [PMID:16966236] http://dx.doi.org/10.1080/09638280600551476

22. Gosney JL, Scott JA, Snook EM, Motl RW. Physical activity and multiple sclerosis: Validity of self-report and objective measures. Fam Community Health. 2007;30(2):144-50. [PMID:19241650]

23. Ng AV, Kent-Braun JA. Quantitation of lower physical activity in persons with multiple sclerosis. Med Sci Sports
Exerc. 1997;29(4):517-23. [PMID:9107635]

http://dx.doi.org/10.1097/00005768-199704000-00014

24. Motl RW, Suh Y, Dlugonski D, Weikert M, Agiovlasitis S, Fernhall B, Goldman M. Oxygen cost of treadmill and over-ground walking in mildly disabled persons with multiple sclerosis. Neurol Sci. 2011;32(2):255-62.

[PMID:20798968]

http://dx.doi.org/10.1007/s10072-010-0396-0

Submitted for publication March 31, 2011. Accepted in revised form October 4, 2011.

This article and any supplementary material should be cited as follows:

Sandroff BM, Motl RW, Suh Y. Accelerometer output and its association with energy expenditure in persons with multiple sclerosis. J Rehabil Res Dev. 2012;49(3): 467-76.

http://dx.doi.org/10.1682/JRRD.2011.03.0063

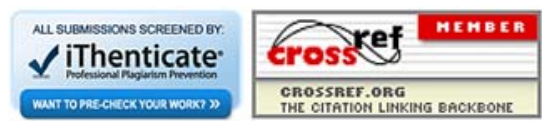


